\title{
Criminologie
}

\section{Carrières criminelles collectives : évolution d'une population délinquante (groupes de motards)}

\author{
Pierre Tremblay, Sylvie Laisne, Gilbert Cordeau, Angela Shewshuck et Brian \\ MacLean
}

Volume 22, numéro 2, 1989

Le milieu criminel

URI : https://id.erudit.org/iderudit/017282ar

DOI : https://doi.org/10.7202/017282ar

Aller au sommaire du numéro

Éditeur(s)

Les Presses de l'Université de Montréal

ISSN

0316-0041 (imprimé)

1492-1367 (numérique)

Découvrir la revue

Citer cet article

Tremblay, P., Laisne, S., Cordeau, G., Shewshuck, A. \& MacLean, B. (1989). Carrières criminelles collectives : évolution d'une population délinquante (groupes de motards). Criminologie, 22(2), 65-94.

https://doi.org/10.7202/017282ar
Résumé de l'article

Co-offending has not been a major area of research for students of crime careers. This paper, however, offers a preliminary analysis of an extensive set of intelligence files gathered by law enforcement agencies on biker groups over a 14 year period (1974-1988). Data has been collected on size, location, network status, life span and degree of crime involvement of 62 criminally involved adult biker groups having operated in Eastern Canada (Quebec) during that period. Findings show a substantial drop in participating groups. Further, remaining groups have not become larger as shown by the equally substantial drop in the overall core underworld biker population. Alternative explanatory accounts for this overall drop are considered. 


\title{
CARRIÈRES CRIMINELLES COLLECTIVES: ÉVOLUTION D'UNE POPULATION DÉLINQUANTE (LES GROUPES DE MOTARDS)।
}

Pierre Tremblay ${ }^{2}$, Sylvie Laisne ${ }^{3}$, Gilbert Cordeau ${ }^{4}$, Brian MacLean ${ }^{5}$, Angela Shewshuck6

\begin{abstract}
Co-offending has not been a major area of research for students of crime careers. This paper, however, offers a preliminary analysis of an extensive set of intelligence files gathered by law enforcement agencies on biker groups over a 14 year period (1974-1988). Data has been collected on size, location, network status, life span and degree of crime involvement of 62 criminally involved adult biker groups having operated in Eastern Canada (Quebec) during that period. Findings show a substantial drop in participating groups. Further, remaining groups have not become larger as shown by the equally substantial drop in the overall core underworld biker population. Alternative explanatory accounts for this overall drop are considered.
\end{abstract}

La criminalité est dans une large mesure une activité grégaire: les sondages de victimisation indiquent que les deux tiers des délinquants (adultes ou juvéniles) commettent leurs crimes soit par groupes de deux ou trois, soit par groupes de quatre individus ou plus (Reiss, 1980; 1986). Toutefois il est généralement admis que la densité sociale des populations délinquantes (l'étendue, l'intensité et la durée des interactions interindividuelles) est faible. La grande majorité des associations dyadiques qui s'établissent entre délinquants se dissolvent après six mois (Sarnecki, 1986); le pourcentage de délinquants affiliés à des bandes structurées est relativement faible (10 à $12 \%$ selon les travaux

1. Cette recherche a été subventionnée à la fois par le Conseil de recherche en sciences humaines du Canada et par le F.C.A.R. Nous voudrions remercier Jacques Cloutier, Raymond Dorval, Jérome Denis et Pierre Fréchette, ainsỉ que le lieutenant Duchesne de la Sûreté du Québec pour leur collaboration précieuse. Nous remercions également Monica Kipiniak pour son aide et Pierre McDuff pour avoir réalisé le traitement initial des archives policières disponibles.

2. Professeur au Département de sociologie, Université McGill; chercheur associé au Centre international de criminologie comparée de l'Université de Montréal, C.P. 6128, Succ. A, Montréal, H3C 3J7.

3. Agent de recherche, Centre international de criminologie comparée de l'Université de Montréal.

4. Agent de recherche, Centre international de criminologie comparée de l'Université de Montréal.

5. Auxiliaire de recherche, Département de sociologie, Université McGill.

6. Auxiliaire de recherche, Département de sociologie, Université McGill. 
recensés par Stafford, 1985), et les bandes dont l'espérance de vie dépasse une année sont fort rares (Suttles, 1968; Sarnecki, 1986). La taille des groupements délinquants quoique variable est généralement limitée: la centaine de bandes de délinquants juvéniles étudiées en Suède par Sarnecki (1986) étaient composées en moyenne de 5 membres et les 32 bandes («street corner gangs») observées à Chicago par Suttles (1968) en regroupaient une douzaine. Lorsque les réseaux d'associations semblent plus étendus, une analyse plus fine découvre plutôt un tissu poreux et un sous-ensemble de "cliques» actives (de 5 à 10 membres) autour desquelles gravite un ensemble beaucoup plus nombreux d'individus dont la participation apparaît comme très occasionnelle, passive et éphémère (Klein et Crawford, 1967).

Dans cette perspective, et comme l'a souligné Horowitz (1982), les mérites théoriques d'une analyse plus élaborée des groupes de motards délinquants sont d'autant moins négligeables que de tels groupes constituent par leur longévité et l'âge moyen de leurs membres (la trentaine aujourd'hui) une sorte d'anomalie ou d'improbabilité conceptuelle pour une sociologie criminelle dont la principale tâche, semble-t-il, a été de rendre compte de diverses manières de la labilité intrinsèque des associations délinquantes, et de l'instabilité plus marquée encore de ces groupes pour qui le courage physique, l'honneur et la pratique de la violence représentent une préoccupation centrale (Yablonsky, 1963).

En reconstituant la carrière collective d'un réseau en quelque sorte naturel d'activistes criminels, il devrait être possible également de combler une lacune des recherches existantes en matière de carrières criminelles (pour un bilan récent de ces travaux initiés par Wolfgang et al., $1972 \mathrm{cf}$. LeBlanc, 1987). Bien que l'on admette que les délinquants affiliés ou disposant d'un large réseau de complices développent une carrière criminelle plus intense, plus sérieuse et plus durable (Curry et Spergel, 1988; Reiss, 1986), la plupart des recherches longitudinales mises en place pour suivre la trajectoire de cohortes délinquantes ont négligé de tenir compte de ce facteur crucial : en procédant par échantillonnage aléatoire, chaque sujet des cohortes ainsi étudiées se voit par définition extrait et aliéné de son réseau local d' «associations différentielles». Jusqu'à quel point une carrière criminelle constitue une trajectoire nodale où s'entrecroisent les carrières d'un nombre variable de coparticipants successifs et jusqu'à quel point ces interactions permettraient rétroactivement de comprendre plus finement les séquences délinquantes individuelles - ces questions sont évacuées d'office. Le 
«vice de forme» est d'autant plus problématique que ces travaux ont pour objectif principal d'identifier et de reconstituer la dynamique qui soustend les carrières des délinquants «chroniques».

Pour finir, mentionnons que les monographies détaillées sur groupes de motards criminalisés sont fort rares bien qu'il s'agisse de groupes exotériques (insignes, locaux, etc.), et beaucoup plus facilement repérables que les sociétés ésotériques auxquelles on identifie le milieu criminel (à tort, cependant, comme l'a montré Reuter, 1983). Exception faite d'un classique littéraire mineur sur le plus notoire de ces groupes de motards, les Hell's Angels (Thompson, 1968), la seule enquête solide dont nous disposons a été menée par un journaliste qui a entrepris de reconstituer l'histoire d'un groupe de motards ontarien, les Satan's Choice, typique des années 1968-74 (Lowe, 1988). Bien que les groupes de motards contemporains constituent une forme bien typée fortement communale d'association délinquante adulte, les travaux dans ce domaine sont extrêmement rares. L'observation participante, une stratégie de recherche souvent adoptée lorsqu'il s'agit d'étudier les bandes de délinquants juvéniles, ne convient guère: les observateurs semi-institutionnalisés que sont les travailleurs de rue disparaissent lorsque la délinquance cesse d'être juvénile et territorialisée dans les zones socialement désorganisées ou les ghettos des métropoles. Les rares enquêtes sur le terrain qui se soient intéressées aux groupes relativement stables de délinquants adultes ont changé de perspective, le milieu carcéral prenant le relais des ghettos comme site privilégié d'observation des gangs et des super-gangs (Jacob, 1974; 1976; 1977; 1979).

Dans cette étude, l'enquête de terrain est délaissée au profit d'une analyse des archives policières. Au lieu d'une monographie détaillant les carrières individuelles des membres d'un groupe particulier, nous proposons de reconstituer la trajectoire collective, depuis 15 ans, d'une population délinquante prise dans son ensemble (62 groupes de motards). L'article est divisé en trois parties. La première section, principalement méthodologique, décrit les archives analysées, les tests de validation utilisés et les critères choisis pour reconstituer le sousensemble des groupes de motards que l'on peut considérer comme criminellement actifs. La deuxième partie examine les phases successives d'expansion et de contraction qui ont modulé l'évolution de ces groupes. La troisième section, beaucoup plus spéculative, examine diverses hypothèses susceptibles de rendre compte de la contraction des effectifs que l'on observe au début des années 1980. 


\section{DONNÉES}

Si les statistiques criminelles contemporaines conviennent pour une théorie des mouvements généraux de la criminalité de masse (Gove et al., 1987), l'analyse des pratiques criminelles ou de populations délinquantes spécifiques - province privilégiée de ce que l'on appelle parfois la criminologie spéciale - explore plutôt les archives confidentielles que tiennent à jour les unités d'enquête spécialisées ou les services de renseignements criminels. Ainsi, les dossiers thématiques qu'il a été possible de consulter sur un ensemble initial de 151 groupes de motards établis dans la province de Québec et actifs durant les années 1973-1988 (corpus documentaire $\mathrm{n}^{\circ} 1$ et sphère privilégiée des renseignements criminels) permettent, dans un deuxième temps, de retrouver les feuilles de route, c'est-à-dire les carrières pénales individuelles des membres identifiés (corpus documentaire $n^{\circ} 2$ et domaine hautement centralisé des services d'identité judiciaire), et ces antécédents criminels conduisent à leur tour à la masse des dossiers d'enquête ponctuels relatifs aux délits particuliers dont ceux-ci ont été accusés ou condamnés (corpus $n^{\circ} 3$ et terrain beaucoup plus diversifié des escouades spécialisées d'enquête). Lorsqu'il s'agit de rendre compte des conditions de possibilité d'une pratique criminelle donnée, l'exploration des archives peut être qualifiée d'ascendante (corpus $3 \rightarrow$ corpus $2 \rightarrow$ corpus $\rightarrow 1$ ). Lorsque l'objet d'étude est une population délinquante préalablement définie, le cheminement documentaire procède en sens inverse (corpus $\rightarrow 1$ corpus $\rightarrow 2$ corpus $\rightarrow 3$ ). L'analyse de ces trois niveaux d'archives déborde le cadre de cet article; nous traiterons surtout des dossiers thématiques, notre tâche liminaire étant de reconstituer en premier lieu les règles généalogiques implicites qu'une telle définition constitutive (la population des motards délinquants) présuppose.

Trois règles ont été suivies pour délimiter la base empirique de cette étude.

a) Le recensement se limite aux groupes ou chapitres de motards dont les locaux sont établis dans la province de Québec. Une territorialisation partiellement arbitraire compte tenu de la mobilité géographique des motards ou des interactions parfois étroites que certains groupes peuvent entretenir avec les groupes établis officiellement en Ontario ou en Nouvelle-Angleterre. En pratique, cependant, il s'agit d'un territoire naturel d'analyse pour une étude préliminaire.

b) Deuxièmement, seules les archives des renseignements criminels de la Sûreté du Québec ont été traitées. Il eût été préférable, sans doute, de les coupler aux renseignements dont disposaient d'autres 
corps policiers, mais la dispersion géographique de ces groupes et de leurs chapitres dans plus de 200 municipalités ou localités (cf. annexe), à l'écart souvent des principales métropoles urbaines, explique en partie la sorte de monopole que ce service détient en la matière. En outre, il existe souvent une division informelle de travail pratiquée par les corps policiers dont les juridictions se chevauchent ou se complètent, les services de renseignements de la police provinciale jouant en l'occurrence le rôle d'instance de coordination.

c) La période d'observation se limite principalement aux années 1973-1988 pour diverses raisons. Bien que les groupes de motards délinquants aient fait leur apparition au milieu des années soixante alors que s'amorçait le début de la plus grande vague de criminalité que les pays occidentaux ont connue depuis la fin du $19^{\mathrm{e}}$ siècle (Gurr, 1977), ce ne sera qu'une dizaine d'années plus tard qu'ils feront l'objet d'une préoccupation publique clairement formulée avec la création en 1973 d'une escouade anti-motards par les services de renseignements criminels de la police provinciale ontarienne (Lowe, 1988, p. 112). Évolution similaire des services de renseignements de la Sûreté du Québec, la létalité des conflits qui opposèrent en 1974 et en 1975 les Popeyes, les Devils Disciples et les Satan's Choice (18 motards ont été alors victimes de règlements de comptes) servant de catalyseur. Les audiences publiques de la Commission d'enquête sur le crime organisé ${ }^{7}$ furent consacrées en 1978 et en 1979 à certains groupes délinquants de motards et la Sûreté du Québec réalisa à cette occasion une sorte de recensement maison auquel participèrent l'ensemble des corps policiers municipaux de la province: c'est ce recensement de 1978 (mis à jour périodiquement par la suite) qui a permis de monter les 151 dossiers thématiques que nous avons consultés.

\section{CRITÈRES DE SÉLECTION}

Ces dossiers étant de valeur et d' «épaisseur» inégales (certains d'entre eux ne mentionnent qu'une série laconique de noms ou prénoms, alors que d'autres contiennent jusqu'à 8000 pages de notes, transcriptions et rapports divers), il était préférable de reconstituer un

7. Les travaux de la CECO (1980) ont porté sur les activités de 12 des 38 groupes actifs en 1978. Près de 300 témoins ont été entendus lors des audiences de la Commission. Celle-ci a mobilisé la mise en accusation de 41 motards (30 furent subséquemment condamnés). Ces poursuites n'ont contribué à la dissolution que d'un seul de ces groupes, les Black Spiders de Saint-Michel-de-Bellechasse, qui se sabordèrent en 1979 suite à la condamnation de 13 membres. Parmi ceux-ci, 9 furent condamnés à des sentences carcérales d'une durée moyenne de 5 ans. Fait à retenir, la Commission ne s'intéressa ni aux Hell's Angeis ni aux Outlaws. 
champ documentaire moins étendu mais plus homogène. La procédure de sélection a été principalement négative. Certains groupes n'ont pas été retenus parce qu'il n'existait aucune information précise sur le groupe lui-même (sa création, sa dissolution, ses affiliations, etc.) ou sur ses membres (simple liste énumérative de certains prénoms ou noms); ou encore parce que les informations disponibles ne mentionnaient aucune participation à une activité criminelle particulière de la part des membres présumés.

Parmi les 150 groupes fichés 8 seulement 63 ont été retenus pour l'analyse: $70 \%$ des 1530 membres identifiés de ces groupes (dont la liste est fournie en annexe) possèdent des antécédents judiciaires et $42 \%$ ont connu, au moins une fois au cours de leur carrière pénale, une sentence carcérale de 3 mois et plus. Les feuilles de route des membres identifiés d'un échantillon de 22 des 87 groupes exclus de l'analyse sont beaucoup moins éloquentes: seulement $10 \%$ des 101 membres identifiables furent condamnés au moins une fois à une sentence d'emprisonnement de 3 mois ou plus.

Il est difficile, compte tenu de l'état relativement peu avancé de nos travaux, d'estimer jusqu'à quel point cet ensemble de 1530 motards identifiés constitue un échantillon représentatif de la population des motards qui ont été criminellement actifs durant les années 1970-1980. De plus, la probabilité que ces activistes aient été fichés par les services de renseignements n'a probablement pas été la même tout au long de la période considérée. On peut supposer, en fait, que la surveillance des corps policiers devint beaucoup plus rigoureuse après 1977 , lorsque la CECO reçut le mandat officiel de se pencher sur cette variété bruyante de délinquants, et que par conséquent le chiffre «noir» des motards criminellement actifs, mais absents des registres des services de renseignements, fut plus important avant 1978. On peut le vérifier indirectement. Nous disposons d'un recensement, à toutes fins pratiques exhaustif, des règlements de comptes commis au Québec de 1970 à 1986 (Cordeau, 1989) et il a été possible de déterminer que $118(13,7 \%)$ des 859 «participants» à ces homicides (victimes ou suspects présumés) étaient des membres de groupes de motards ${ }^{9}$. Le recoupement de ces deux banques de données est instructif. De 1970 à 1986, $60 \%$ des

8. Chaque groupe disposant de 1,4 chapitre en moyenne, les 150 groupes représentent 212 sous-ensembles distincts de motards.

9. Ce recensement s'appuie sur les archives d'un hebdomadaire spécialisé dans l'homicide (Allô Police) et sur les rapports d'enquête des escouades spécialisées de la Sûreté du Québec et du Service de police de la Communauté urbaine de Montréal. La méthodologie utilisée est décrite dans l'article de Gilbert Cordeau inclus dans ce numéro. 
motards impliqués dans ces conflits endogènes au milieu criminel faisaient partie des groupes que nous avons retenus pour analyse et de la population des motards identifiés dont nous connaissons les antécédents judiciaires. Un pourcentage un peu décevant, mais ce pourcentage est de $75 \%$ pour les règlements de comptes commis de 1978 à 1986 et de $37 \%$ seulement lorsqu'ils ont eu lieu entre 1970 et 1977.

\section{VALIDITÉ GÉNÉRALE DES DONNÉES}

Il est toujours possible, bien entendu, de supposer que la cueillette des renseignements effectuée par les corps policiers et centralisée aux services de renseignements criminels se soit constituée de manière anarchique et désordonnée. Si tel est le cas on s'attendrait à ce qu'il n'existe guère de rapport entre le nombre de membres fichés associés à tel ou tel groupe de motards surveillé par les corps policiers et les caractéristiques réelles du groupe lui-même. L'hypothèse alternative soutiendrait au contraire que la probabilité pour qu'un motard ou un groupe de motards soit fiché par les renseignements criminels dépendrait à la fois de sa taille, de son degré d'engagement criminel et de sa longévité. Mentionnons aussi que n'importe quelle vérification routière permet d'obtenir le nom complet, la date de naissance d'un individu et ses autres coordonnées fondamentales de sorte que l'absence de tels renseignements témoignerait du peu d'intérêt que les corps policiers portent au groupe fiché. Afin d'obtenir une mesure relativement indépendante de ces trois facteurs (taille, longévité et réputation criminelle), nous avons demandé à certains «connaisseurs» 10 d'examiner la liste des

10. Quatre des 8 motards ou ex-motards sollicités ont accepté de remplir le questionnaire. Les répondants ont en moyenne 35 ans et étaient membres de groupes de motards opérant dans la région de Montréal. Bien qu'ex-membres ou anciens membres de groupes dissous, ils sont encore actifs dans le milieu et leur statut actuel et passé permet de les localiser a la périphérie immédiate des groupes de motards que nous analysons dans cet article. Le nombre de refus, assez élevé, peut être lié au fait que les quatre autres contacts sollicités sont membres de groupes encore très actifs. Notons également que même les répondants qui ont accepté de remplir le questionnaire ont refusé d'indiquer l'état actuel des affiliations ou interactions entre les divers groupes de motards sélectionnés pour le questionnaire. Bien qu'il eût été préférable d'obtenir les opinions d'un plus grand nombre d'«experts», l'investissement que requiert une telle enquête de terrain (comme en témoigne par exemple la monographie de Lowe, 1988) était prohibitif, compte tenu du temps disponible. Toutefois les questionnaires complétés permettent d'établir, au moins à titre indicatif, si les données «naturelles» corroborent ou non les données policières. 
groupes de motards retenus pour cette étudelt et d'indiquer pour chaque groupe connu, la taille, l'année de la création, l'année de la dissolution et la réputation criminelle. Ces coordonnées temporelles ont permis de classer les groupes en trois classes, selon leur espérance de vie : brève ( 6 ans et moins), moyenne ( 7 à 12 ans) et élevée (13 ans et plus). Pour simplifier l'entrevue, les répondants devaient également indiquer s'il s'agissait d'un groupe qu'ils considéraient comme moyen, important ou petit, et nous avons retenu comme critère numérique les catégories proposées par les policiers consultés (20 membres actifs ou moins, entre 20 et 40 membres, plus de 40 membres). Quant à la réputation criminelle du groupe, ils devaient nous indiquer s'ils les considéraient comme étant «très actifs», «peu ou pas actifs», ou «moyennement actifs». De manière quelque peu arbitraire, nous avons attribué à chaque groupe de motards un score sommatif global de 3 à 9 , chaque variable ayant trois valeurs et recevant le même poids relatif ${ }^{\prime 2}$.

Pour finir, nous avons examiné le nombre de membres fichés par groupe par les services de renseignements criminels au cours de la période analysée (1974-1988 principalement). Là encore, ces groupes ont été classés en trois sous-ensembles selon que le nombre d'individus identifiés par groupe au cours de l'ensemble de la période était inférieur à 20 , de 20 à 40 ou supérieur à 40 . Le tableau 1 examine jusqu'à quel point le nombre de membres fichés et identifiés par groupe dépend des caractéristiques réelles du groupe telles qu'évaluées par les motards interrogés.

11. L'intervieweur présentait aux répondants cette liste comme étant le résultat de ses propres souvenirs et contacts personnels. L'intervieweur disposait d'ailleurs des «lettres de créance» nécessaires pour ce genre de travail de terrail puisqu ii avait luimême été condamné à plusieurs reprises, notamment pour trafic de stupéfiants, et avait également purgé une sentence pénitentiaire. Ses activités passées l'ont mis en relation avec un bon nombre de groupes de motards et au moment des entrevues il travaillait (a temps partiel) pour le compte d'une firme spécialisée dans la vente d'accessoires de moto, qui participe sur une base régulière aux foires commerciales organisées par les principaux producteurs de moto et auxquelles assistent non seulement les divers clubs sportifs mais aussi les groupes de motards étudiés dans cette recherche. Ces exhibitions sont également surveillées par les corps policiers.

12. Ainsi, un score de 9 signifie que les répondants considèrent ce groupe comme éphémère, peu actif criminellement et de taille réduite. Un score de 3 signifie que les répondants considèrent ce groupe comme étant impliqué d'une façon très active dans le crime, de taille supérieure à la moyenne et ayant une longévité également supérieure à la moyenne. 


\section{TABLEAU 1}

Corrélation entre le nombre de membres identifiés par groupe (par les renseignements criminels) et la cote attribuée à ces groupes par les motards interviewés sur la base de leur taille estimée, leur longévité et leur degré d' activité criminelle perçue

\begin{tabular}{lccc}
\hline & \multicolumn{3}{c}{ Nombre de membres fichés par groupe } \\
$\begin{array}{l}\text { Scores attribués } \\
\text { par les répondants } \\
\text { aux groupes }\end{array}$ & $\begin{array}{c}\text { Groupes de } \\
1 \text { à } 19 \\
\text { membres }\end{array}$ & $\begin{array}{c}\text { Groupes de } \\
20 \text { à } 39 \\
\text { membres }\end{array}$ & $\begin{array}{c}\text { Groupes de } \\
40 \text { membres } \\
\text { et plus }\end{array}$ \\
\hline Scores 7-9 & 20 & 6 & 3 \\
Scores 5-6 & 5 & 6 & 2 \\
Scores 3-4 & 0 & 1 & 6 \\
\hline
\end{tabular}

Taux $\mathrm{c}=, 43$

Coefficient de contingence $\mathrm{C}=, 57$

Indice d'efficacité prédictive: $42 \%$

Les résultats sont passablement encourageants. II existe une association substantielle entre les scores sommatifs attribués par les répondants aux groupes de motards évalués et le nombre de membres par groupe fichés et identifiés par les services de renseignements criminels (Taux c de ,48 et coefficient de contingence de ,53). L'indice d'efficacité prédictive mis au point par Ohlin et Duncan en 1949 (Simon, 1971) est également substantiel ( $42 \%$ ) et sa valeur deux fois plus élevée que si l'on utilisait la taille estimée du groupe comme seul prédicteur. Cet indice, similaire à «l'indice d'amélioration relative du hasard» utilisé par exemple par Loeber et Loeber (1986), signifie simplement que si l'on voulait prédire le nombre moyen de membres fichés par groupe par les renseignements criminels, une bonne manière d'éviter de commettre des erreurs serait de connaître la taille réelle du groupe, mais qu'une manière encore plus efficace de réduire nos erreurs serait de tenir compte également de sa «réputation criminelle» et de sa «longévité». Ces résultats n'ont rien de particulièrement transcendant mais indiquent seulement que les archives de renseignements consultées ne se sont pas élaborées au hasard mais en fonction de la persistance et du degré d'engagement criminel des groupes surveillés et que, de ce point de vue, la population délinquante que nous étudions ici est raisonnablement bien définie. 


\section{CONTRACTION D'UNE POPULATION DÉLINQUANTE ADULTE}

Les carrières collectives particulières des divers groupes de motards permettent de reconstituer la trajectoire globale de l'ensemble de cette population délinquante adulte. La chronologie que nous proposons se base à la fois sur les dates d'incorporation de ces groupes comme clubs sportifs, les informations consignées dans les archives policières consultées et les estimations que les motards interrogés nous ont fournies. Aucune de ces trois sources de renseignements n'est suffisante en elle-même. Même si bon nombre de ces groupes se sont incorporés comme clubs sportifs au Fichier central des entreprises du Québec, la date d'enregistrement permettant de dater le début de leur existence «publique» (ainsi les Primitifs de Beauharnois se sont incorporés en 1974, les Missiles de Baie Comeau en 1978 et les Sex Fox de Chibougamau en 1976), d'autres ne l'ont pas fait (par exemple les Atomes de Rock Forest ou les Z'Beers et les Dragons de St-Hyacinthe). Il arrive souvent que l'année de formation du groupe précède l'année officielle d'enregistrement (ainsi la charte fédérale des Hell's Angels date de 1979, mais la formation dans la province de Québec des chapitres de ce groupe remonte à 1977). Et le Fichier central des entreprises du Québec n'est guère utile pour déterminer l'année de dissolution du groupe. Les archives policières, de leur côté, sont souvent précieuses, mais elles se sont élaborées progressivement et les dossiers thématiques sur les groupes eux-mêmes sont moins complets lorsqu'il s'agit de reconstituer les coordonnées des groupes des années 60 ou du début des années 70 . Finalement, les dates de création ou de dissolution proposées par les motards interrogés permettent de compléter certaines lacunes mais ces estimations ne sont pas toujours concordantes, d'où la nécessité de procéder par interpolation. Il en résulte que la chronologie que nous avons établie par recoupement, corroboration et interpolation des sources d'information disponibles est intrinsèquement approximative. II serait peut-être même vain d'ailleurs de songer à une chronologie rigoureusement précise et objective puisque nous nous intéressons moins à l'histoire privée de ces groupes (aux trajectoires particulières de ceux qui les fondèrent) qu'à leur carrière publique, au moment de leur formation comme groupes délinquants «reconnus» à la fois par les autres groupes de motards et par les corps policiers.

On trouvera en annexe la chronologie des carrières particulières de chacun de ces groupes et leur espérance de vie (en moyenne 9,6 années). Il va sans dire que certains groupes sont relativement éphémères (les «Québécois» de Coaticook ont existé pendant moins de 
TABLEAU 2

Création, dissolution et persistance des groupes de motards criminellement actifs (Province de Québec, 1969-1988)

\begin{tabular}{lccccc}
\hline & & $\begin{array}{c}\text { Groupes qui se } \\
\text { sont dissous } \\
\text { durant la } \\
\text { période t* }\end{array}$ & $\begin{array}{c}\text { Groupes } \\
\text { formés en t* }\end{array}$ & $\begin{array}{c}\text { Groupes opérant } \\
\text { en t-i et t }\end{array}$ & TOTAL \\
\hline $1969-1972^{* *}$ & & & & & \\
$1973-1977$ & $\mathrm{~N}$ & 3 & 11 & 27 & 41 \\
& $\%$ & $7 \%$ & $27 \%$ & $66 \%$ & $100 \%$ \\
$1978-1983$ & $\mathrm{~N}$ & 26 & 15 & 17 & 58 \\
& $\%$ & $45 \%$ & $26 \%$ & $29 \%$ & $100 \%$ \\
$1984-1988$ & $\mathrm{~N}$ & 18 & 1 & 14 & 33 \\
& $\%$ & $55 \%$ & $3 \%$ & $42 \%$ & $100 \%$ \\
\hline
\end{tabular}

* Les groupes éphémères et dont la carrière «ponctuelle» se limite à une seule période sont comptés deux fois, à la fois comme groupes «dissous» et comme groupes «formés": c'est le cas de 6 groupes en 1973-1977.

** Les années 1969-72 servent de période de référence. Parmi les 34 groupes de motards recensés durant ces trois années, 30 se sont formés durant ces années, 2 datent d'avant 1969 et 2 se sont dissous avant 1973. Ce recensement est sans doute incomplet, particulièrement en ce qui concerne les dissolutions.

quatre années) alors que d'autres témoignent d'une longévité singulière (les «Vikings» de Granby et de Matane survivront 15 ans et les «Atlans» de Québec existent encore après 17 ans de loyaux services). La capacité de renouvellement que manifeste cette population délinquante est également variable de sorte que le nombre de groupes qui existent simultanément durant une année donnée ( 28 environ) varie également au cours de la période (38 groupes en 1977 mais seulement 15 en 1987). Le tableau 2 décrit, par tranche de cinq ans, les principales phases de recomposition successives que cette population délinquante a traversées depuis 20 ans.

Bien que les groupes ayant survécu tout au long de la période soient peu nombreux (les «Blatnois» de St-Wenceslas ou les «Christ Drivers» de Senneterre), on peut noter que, pour chaque phase, au moins le tiers des groupes observés maintiennent une carrière collective suffisamment longue pour chevaucher deux intervalles consécutifs (1969-72 et 1973-77; $1973-77$ et 1978-82, etc.). Un indice passablement patent de la capacité pour cette population de développer une «tradition», capacité d'autant plus remarquable qu'elle infirme les 
conclusions fort pessimistes auxquelles parvient la sociologie contemporaine des bandes de délinquants juvéniles et dont la thèse centrale, rappelons-le, fut de souligner leur foncière instabilité.

La capacité de cette population délinquante de se renouveler est particulièrement marquée de 1973 à 1977 comme en témoigne le fait que le taux de recrutement est largement supérieur au taux de dissolution. Une phase d'expansion, par conséquent, et il est probable d'ailleurs qu'il s'agisse d'un processus amorcé dans les années 1965 et que les années 1973-77 en marquent la fin (cf. Lowe, 1988, p. 83). Durant les années 1978-82, par contre, le scénario s'inverse et les «abandons» sont plus fréquents que les «créations»: donc, une phase de recomposition qui, soit dit en passant, marque également l'implantation officielle (en 1977), dans la province de Québec, des grands «clubs» américains.

Durant les années 1983-88, le nombre de groupes baisse substantiellement. Les nouveaux groupes qui se forment sont très rares (les «Warriors» de Boisbriand) et un grand nombre d'entre eux disparaissent. Il est possible que les groupes qui survivent durant ces années soient moins nombreux mais qu'ils disposent d'un cercle élargi de membres. D'où l'intérêt d'estimer, sur une base annuelle, le taux dé participation global à ces groupes pour l'ensemble de la période. La figure 1 résume les estimations faites par les motards interviewés et celle proposée par un des officiers des services de renseignements.

FIGURE 1

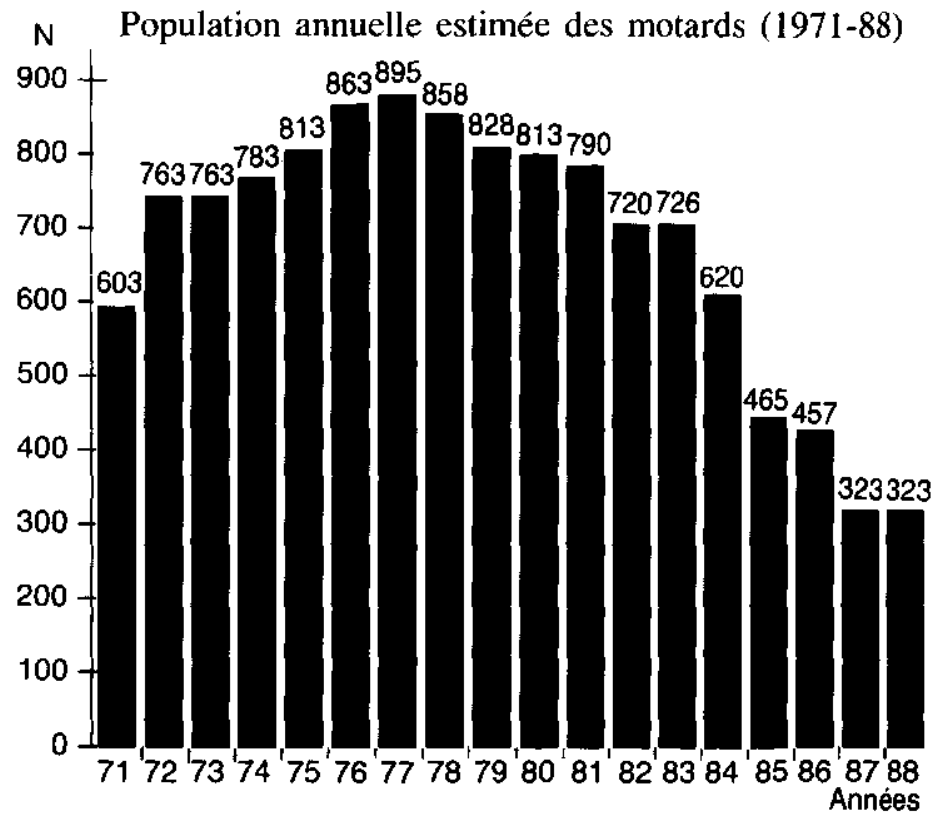


Chaque répondant devait classer les groupes en trois catégories selon leur taille approximative (petit groupe: moins de 19, groupe moyen : 20 à 39 et groupe important : 40 et plus). La «population estimée de motards» a été établie annuellement en faisant la somme pondérée des membres attribués à chacun des groupes en opération durant l'année d'observation (les facteurs de pondération étant respectivement de 10 pour les petits groupes, de 30 pour les groupes moyens et de 50 pour les groupes majeurs). Lorsque les estimations des divers répondants ne concordaient pas, nous avons procédé par interpolation. Il peut être opportun de noter que la plupart des répondants nous ont objecté que les catégories qualitatives utilisées (petit groupe vs gros groupe) n'avaient pas la même signification selon qu'il s'agissait de la fin des années 1960 (où les gros groupes disposaient souvent d'une centaine de membres et plus) ou du début des années 1980 (où l'on considère un groupe de $\mathbf{4 0}$ membres comme majeur. La figure 1 sous-estime aussi, fort probablement, la population annuelle de motards au début des années $1970^{13}$. Et du même coup, sous-estime la baisse tendancielle du taux de participation aux groupes de motards criminellement actifs. Par ailleurs, il est tout à fait improbable que cette contraction puisse être imputable à une sorte d'indifférence progressive des corps policiers à leur égard: leur surveillance s'est au contraire systématisée à partir de $1978^{14}$. La section suivante examine les diverses hypothèses susceptibles de rendre compte de cette évolution collective.

\section{GAINS À LA BAISSE, COÛTS À LA HAUSSE}

Deux séries d'hypothèses peuvent être invoquées à ce sujet. Bien que chacune des hypothèses que nous examinerons possède sa propre niche théorique, on peut considérer de manière générale que la première série d'explications attribue ce déclin aux avantages ou bénéfices décroissants que les délinquants peuvent retirer de leur appartenance à ces groupes. La seconde série d'hypothèses soutient au contraire que les coûts croissants d'une telle participation constituent le facteur crucial et que la contraction des «effectifs» ne témoignerait pas tant d'un déclin ou d'une décadence mais d'un processus de sélection ou d'épuration. Précisons qu'il ne s'agit pas ici de procéder à une vérification d'hypothèses stricto sensu ou de proposer une explication empiriquement établie, mais d'en spécifier les conditions de possibilité.

13. Cette sous-estimation est peut-être moins frappante qu'elle n'y paraît à première vue : il est généralement admis que les groupes d'une centaine de membres et plus sont en réalité composés de sous-cliques de 30 à 40 individus autour desquelles gravitent des membres occasionnels et passifs (Klein et Crawford, 1967),

14. Cf. infra p. 8 
Une première hypothèse peut être qualifiée de culturelle : elle suppose que l'attrait que présente le style de vie des motards (un style qu'ont décrit Thompson en 1965 et Montgomery en 1976 et 1977) se soit estompé au profit d'autres styles de vie ou de modèles de rébellion. Il est possible notamment que le caractère communal ou sectaire de ces groupes n'attire plus qu'une minorité de plus en plus marginalisée de délinquants potentiels. La contraction des effectifs annuels résulterait ainsi d'une difficulté croissante à recruter des candidats. Une hypothèse difficile à prouver directement et qui déborde tout à fait le cadre de cette analyse. Elle exigerait que nous puissions décrire avec précision les caractéristiques particulières du style de vie des motards et trouver dans les journaux, dans la littérature ou dans les sondages, une manière de mesurer la popularité décroissante de styles de vie, d'idéologies ou de modes présentant des affinités marquées avec la sorte de rébellion pratiquée par les motards. Une mise à l'épreuve indirecte de cette explication est toutefois possible. Si le déclin de la population des motards résultait d'une sorte de désaffection généralisée à l'égard du style de vie qu'ils pratiquent, on devrait s'attendre à son vieillissement progressif, les recrues potentielles se tournant plutôt vers d'autres styles de vie ou de rébellion culturellement plus «appropriés».

Une deuxième explication pourrait être également envisagée. Il est possible que les groupes de motards aient été confrontés à une sorte d'appauvrissement progressif de leurs gains illicites ou des opportunités criminelles auxquelles ils avaient accès. On a souvent insisté, par exemple, sur le contrôle monopolistique qu'ils auraient exercé au début des années 1970 sur le marché des drogues chimiques (amphétamines notamment) et il semble que la popularité de cette variété de drogues ait décliné substantiellement. On peut donc supposer qu'un déclin des gains illicites, quelle que soit leur source - le trafic de stupéfiants illicites n'étant mentionné qu'à titre illustratif -, démobiliserait ou démoraliserait de manière plus prononcée les plus âgés des motards: alors que les satisfactions symboliques semblent déterminantes en début de carrière criminelle, elles perdent progressivement, avec l'âge, de leur importance au profit de considérations plus matérialistes (Petersilia et coll., 1977; Peterson et Braiker, 1980). Une telle hypothèse prédirait ainsi une baisse tendancielle de la moyenne d'âge des membres de motards, leur paupérisation collective les incitant à se désister de plus en plus tôt. 
TABLEAU 3

Moyenne d'âge annuelle des motards fichés dans les données policières (1974-1988)

\begin{tabular}{crccc}
\hline Années & $\mathrm{N}$ & Moyenne & Écart type & C.V.* \\
\hline 1974 & 115 & 23,5 & 4,35 & $18,51 \%$ \\
1975 & 56 & 25,0 & 4,96 & $19,84 \%$ \\
1976 & 98 & 25,0 & 4,61 & $18,44 \%$ \\
1977 & 318 & 24,6 & 4,30 & $17,48 \%$ \\
1978 & 289 & 25,4 & 4,97 & $16,02 \%$ \\
1979 & 300 & 25,4 & 4,30 & $16,93 \%$ \\
1980 & 291 & 26,0 & 4,99 & $19,19 \%$ \\
1981 & 185 & 28,0 & 4,43 & $15,82 \%$ \\
1982 & 286 & 26,5 & 5,06 & $19,09 \%$ \\
1983 & 232 & 27,6 & 5,75 & $20,83 \%$ \\
1984 & 235 & 29,0 & 6,11 & $21,07 \%$ \\
1985 & 230 & 30,0 & 5,41 & $18,03 \%$ \\
1986 & 177 & 31,0 & 5,60 & $18,06 \%$ \\
1987 & 76 & 32,0 & 5,60 & $17,50 \%$ \\
1988 & 72 & 31,0 & 5,70 & $18,39 \%$ \\
\hline
\end{tabular}

* Coefficient de variation (écart type / moyenne).

Connaissant à la fois la date de naissance de ces membres et les années durant lesquelles les corps policiers les fichèrent comme tels, il a été possible d'établir l'âge moyen des membres sur une base annuelle de 1974 à 1988. Chaque cohorte annuelle de sujets concerne l'ensernble des vingt à trente groupes de motards actifs durant l'année d'observation. Nous ne savons pas jusqu'à quel point l'échantillon annuel observé est représentatif de l'ensemble de la population de référence mais il est raisonnable de penser que les biais de sélection ne diffèrent pas de manière substantielle d'une année à l'autre.

Comme l'indique le tableau 3 la moyenne d'âge des membres connaît une hausse tendancielle. La constance du coefficient de variation montre qu'il n'y a eu ni resserrement ni amplification des écarts d'âge entre les membres. Par ailleurs, ce qui est vrai de la population des motards en général l'est également de la grande majorité des groupes considérés séparément (cf. annexe pour un profil des moyennes d'âge intra-groupes pour certaines années sélectionnées). Ainsi, les membres des «Atomes» avaient 19 ans en moyenne en 1973, 26 ans en 1979 et 30 ans en 1984. Les «Blatnois» avaient 23 ans en 
1978, 26 ans en 1981 et 30 ans en 1985. Les «Hell's Angels» de 1977 avaient 27 ans et 39 ans en 1988. Et les «Gitans», 23 ans en 1974, 26 ans en 1978 et 35 ans en 1983. En d'autres termes, les écarts d'âge entre groupes ont tendance également à se maintenir. La contraction des effectifs étant associée à une hausse tendancielle de l'âge moyen des membres des divers groupes de motards, l'hypothèse culturelle semble résister mieux à l'examen que l'hypothèse économique. Mais d'autres facteurs sont peut-être tout aussi décisifs.

\section{COÛTS À LA HAUSSE}

Une augmentation marquée, au cours de la période, des risques auxquels furent confrontés les motards participants ou ceux qui songeaient à le devenir, pourrait expliquer, en effet, et tout aussi vraisernblablement, la diminution des effectifs. Si tel était le cas, cette contraction ne devrait pas être interprétée comme un symptôme de marginalisation ou de désorganisation mais comme la résultante d'un processus d'autosélection et d'épuration. Deux sortes de coûts méritent ici d'être pris en considération: les risques de victimisation endogène d'une part (probabilité pour un motard d'être blessé ou tué par d'autres motards ou d'autres participants actifs du milieu criminel), et les risques pénaux d'autre part (probabilité d'être arrêté et incarcéré).

Les sondages de victimisation indiquent que ce sont les délinquants qui ont la probabilité la plus élevée d'être victimisés (Singer, 1981). Une observation fondamentale mais rarement incorporée par la sociologie criminelle, même si les romanciers et la littérature populaire en ont pris conscience depuis longtemps ${ }^{15}$. Les risques de règlements de comptes auxquels les motards ont été exposés entre 1970 et 1986 peuvent être évalués. Sachant que 75 motards connus ont été victimes de règlements de comptes durant cette période ( 4,4 en moyenne annuellement) et compte tenu d'une population annuelle moyenne de 713 motards actif́s (voir les estimations de la Figure 1), on peut estimer que

15. David Goodis par exemple dans un de ses premiers romans : «Depuis le temps, tu devrais connaître les coutumes de cette rue... Tiens, tu veux que je te dresse la liste de tous les mômes et de toutes les filles que tu as connus et qui ont disparu du quartier ? Ce n'est pas qu'ils aient simplement déménagé... c'est qu'ils sont morts" (Street of The Lost, 1952 ; trad. fr. Rue Barbare, 1980, p. 76, Ed. Clancier-Guénaud, Paris). Ou ce passage d'une des chansons de Tom Waits : «so I went back to Omaha/to live with my folks/but everyone I used to know/was either dead or in prison", ("Christmas card from a hooker in Minneapolis», Blue Valentine, 1978, Elektra/Asylum Records, New York). Les deux femmes qui s'expriment ainsi ont 25 à 30 ans et le contexte indique très clairement qu'il ne s'agit pas de mort naturelle. 
la probabilité annuelle moyenne pour un motard de mourir pour cause de règlement de comptes a été de 0,0062 (6,2 victimes pour 1000 motards). Un taux très élevé et qui suggère que les motards méritent effectivement leur réputation de violence et de courage, les risques de règlements de comptes parmi les motards étant au moins six fois plus élevés que les risques auxquels sont exposés les participants «ordinaires» du milieu criminel québécois (tels qu'estimés par Cordeau, 1989) ${ }^{16}$. Comme le montre la figure 2 , les risques varient considérablement d'une année à l'autre, les règlements de comptes survenant par vague. La contraction des effectifs observée au début des années 1980 coïncide avec une vague particulièrement importante de règlements de comptes de 1982 à 1985 . Il est donc possible que l'augmentation des risques personnels d'être sérieusement blessé ou tué ait incité bon nombre de groupes à se saborder, de membres à se désister et qu'elle ait intimidé un sous-ensemble non trivial de recrues potentielles. Par ailleurs l'impact négligeable des 18 règlements de comptes commis en 1974 et en 1975 sur la taille de la population de motards (qui ne cesse d'augmenter durant cette période) mérite d'être souligné. Avant 1978 la plupart des groupes opéraient sur une base indépendante et la moitié des règlements de comptes commis en 1974-1975 résultent d'un conflit interne entre deux factions des «Devil's Disciples» (un groupe montréalais). L'arrivée en 1977 des grands clubs américains (les «Hell's Angels», au Québec principalement et les «Outlaws», en Ontario) ont changé les règles du jeu. Le milieu du motard devient plus dense et $54 \%$ des 59 groupes qui ont opéré depuis 1978 sont considérés par les policiers comme leur étant affiliés ${ }^{17}$. On peut penser que les conflits

16. La probabilité qu'un membre du milieu criminel soit tué en raison de conflits résultant de ses activités illicites est elle-même 133 fois plus élevée, selon Cordeau et Tremblay (1989) que celle auquelle est exposée la population mâle adulte prise dans son ensemble. Le calcul de cette variété particulière de «risques occupationnels» présuppose que nous connaissions au départ la taille de la population du milieu criminel. Deux procédures permettent d'obtenir une telle estimation. La première consiste à considérer, moyennant corrections et spécifications, les détenus pénitentiaires comme un échantillon représentatif d'une population ayant les mêmes caractéristiques mais se trouvant encore en liberté ou dans la rue. C'est la procédure de Chaiken et Chaiken (1980). La deuxième procédure, utilisée par Cordeau (1989) ou Cordeau et Tremblay (1989), consiste à dégager la feuille de route typique des victimes et agresseurs présumés impliqués dans les règlements de comptes, et d'évaluer sur la base d'une cohorte représentative de la population en général, le nombre d'individus ayant des feuilles de route similaires. Tous ces calculs concernent exclusivement les risques de décès imputables aux conflits entre participants du milieu criminel.

17. La notion d'affiliation mériterait d'être explicitée de manière beaucoup plus systématique. Pour l'instant il s'agit d'un terme indéfini que nous empruntons à un officier des renseignements criminels à qui nous avons demandé de classer les groupes de motards sous étude (cf. annexe) en trois catégories, selon qu'ils étaient indépendants ou affiliés à l'un ou l'autre des deux grands clubs américains. 
qui opposèrent les Hell's Angels aux Outlaws, eurent beaucoup plus d'impact parce qu'ils impliquaient à divers degrés plus de la moitié de la population totale des motards ${ }^{18}$.

Il est donc possible que ce ne soient pas tant les gains décroissants ou la marginalisation culturelle du style de vie des motards qui expliquent la baisse des effectifs, mais l'augmentation des coûts personnels associés au fait d'être activement impliqués dans de tels groupes. Les risques étant plus élevés, on assisterait à un processus de sélection naturelle: les plus passifs des membres se désistant, les groupes les moins criminalisés se sabordant, la population annuelle totale des motards criminellement actifs devenant, simultanément, moins nombreuse mais beaucoup plus homogène et composée d'un pourcentage croissant de membres motivés et aguerris.

Mentionnons, pour finir, que les risques de victimisation endogènes ne sont peut-être pas les seuls à avoir augmenté à partir du milieu des années 1970. Les risques d'arrestation et de condamnation dépendent en partie du degré de surveillance exercée par les corps policiers. Or celle-ci, on l'a vu, s'est mobilisée en 1974 (durant la première vague de règlements de comptes) et systématisée au début de 1978 (alors que la CÉCO reçut le mandat d'enquêter publiquement sur les clubs de motards opérant dans la province de Québec). Il est donc vraisemblable qu'une analyse détaillée des casiers judiciaires disponibles montrerait une augmentation du pourcentage de motards incarcérés annuellement entre 1975 et 1988. L'impact de cette neutralisation ou «incapacitation» devra être évalué. Deux scénarios ou deux schèmes interprétatifs pourraient être confrontés et mis à l'épreuve. Le premier scénario suppose que la contraction observée des effectifs est une contraction «subie», imputable à l'impact dissuasif généré par l'augmentation des risques pénaux auxquels sont exposés les motards criminellement actifs. Le deuxième scénario propose à l'inverse, que cette contraction est volontaire plutôt que simplement subie. Les risques pénaux ayant augmenté, les groupes de motards auraient adopté une stratégie défensive consistant principalement à renforcer les liens de solidarité entre membres des groupes et entre les groupes eux-mêmes. La baisse des effectifs ne serait pas tant le résultat de défections que d'exclusions. Le vieillisse-

18. Comme l'indiquait l'un des membres des Satan's Choice interviewés par Mike Lowe «... Even Gary Comeau admits the club world he knew during the seventies has changed forever. Things have gotten a lot heavier out there, no doubt about it. The game isn't played by the same rules any more, hell, it isn't even the same ballpark" (Conspiracy of Brothers, 1989, p. 426) 
FIGURE II

Probabilité $(\times 1,000)$ pour un motard d'être

Prob. $(\times 1,000)$ victime d'un règlement de compte

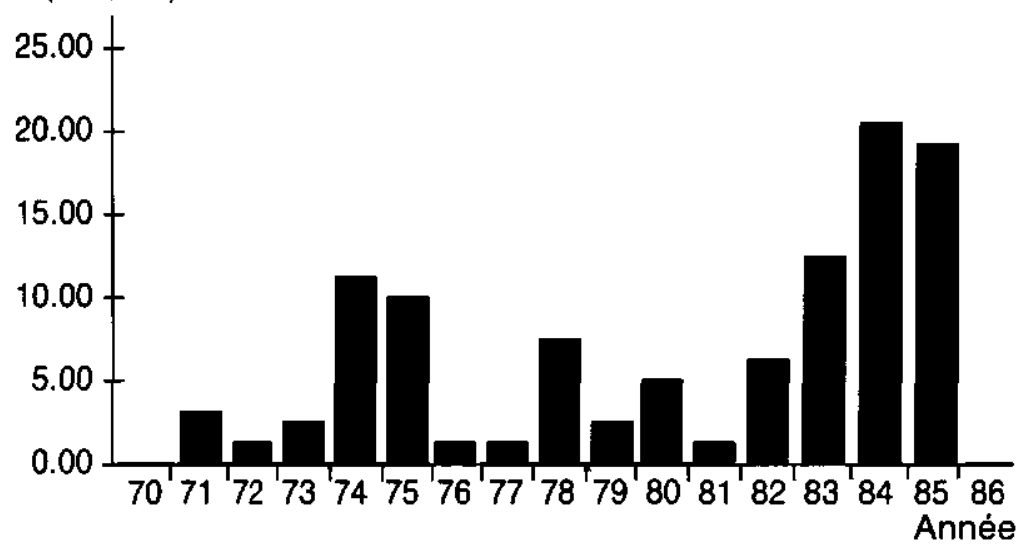

ment de la population des motards témoignerait non pas d'une désaffection des recrues potentielles mais d'un resserrement des critères d'admission. Bon nombre de règlements de comptes ne seraient pas seulement de simples conflits relatifs à la territorialisation des marchés illicites, mais le résultat de mesures disciplinaires destinées à renforcer la cohésion interne des groupes et la densité de la communauté des motards criminalisés dans son ensemble.

\section{CONCLUSION}

On reproche souvent aux analyses exploratoires de se contenter de suggérer ex post facto des hypothèses qu'elles ne soumettent pas à une vérification empirique rigoureuse. Mais comme le notait Liebow (1967), la validité d'une hypothèse ne dépend pas du «timing» de sa formulation originelle mais de sa réplicabilité éventuelle. Par ailleurs, la formulation systématique d'hypothèses alternatives susceptibles de rendre compte d'un fait particulier n'est pas en soi un exercice conceptuel sans mérites, surtout lorsqu'on prend en considération l'anémie analytique qui caractérise selon Stafford (1985) la sociologie des groupes criminels. Les données présentées dans cette étude concernent la trajectoire collective d'un sous-ensemble de groupes de motards dont l'engagement criminel ne fait guère de doute : $70 \%$ des motards identifiés dans cette analyse possèdent un casier judiciaire et $42 \%$ d'entre eux ont connu, au cours de leur carrière pénale, une sentence d'empri- 
sonnement d'une durée moyenne de 4 ans ${ }^{19}$. Par ailleurs la majorité (et après 1977 , les trois quarts) des règlements de comptes recensés pour la province de Québec au cours de cette période et impliquant des victimes ou des suspects identifiés comme des motards (Cordeau, 1989) se retrouvent parmi les groupes retenus dans cette analyse ou parmi les membres dont les feuilles de route sont disponibles.

Premier résultat de cette exploration préliminaire: la longévité remarquable de ces groupes de motards (10 ans en moyenne) et le vieillissement progressif de leurs membres (la trentaine aujourd'hui). Un constat qui remet en cause, au moins indirectement, une des thèses princeps de la sociologie des bandes (juvéniles) délinquantes 20 . Deuxième résultat, là encore de nature quantitative - la contraction de la population annuelle estimée des motards au début des années 1980 , du moins dans le site de recherche considéré (province de Québec). Il est improbable qu'il s'agisse d'un artefact, imputable au déclin de la surveillance exercée par les corps policiers, celle-ci s'étant au contraire systématisée depuis 1977. Pour fins de présentation, une série d'hypothèses relativement distinctes ont été proposées pour rendre compte de cette contraction démographique. La première série d'hypothèses suggère une baisse des gains symboliques (culturels) ou économiques individuels et prédit à terme la marginalisation progressive et la disparition de cette variété particulière de délinquants. La seconde série d'hypothèses, que l'on pourrait qualifier d'optimistes, s'intéresse de manière plus spécifique aux coûts (croissants) associés à cette forme de délinquance et suggère à l'inverse que cette contraction est probablement conjoncturelle et peut-être même temporaire. Cela dit, ces hypothèses ne sont pas mutuellement exclusives: la contraction des effectifs peut être attribuée à la fois à une baisse des gains et à une augmentation des risques (pénaux ou endogènes). Compte tenu du caractère exploratoire de cette recherche, il n'est pas question de trancher entre ces divers scénarios, en partie parce que certaines données n'ont pas été analysées (les feuilles de route des motards fichés par exemple ou la nature exacte des réseaux d'affiliations ou d'interactions privilégiées entre groupes)

19. Les carrières pénales constituent un indicateur acceptable des carrières criminelles proprement dites, comme l'ont montré Blumstein et coll. (1986).

20. Il est vrai que nous ne connaissons pas le roulement interne des membres. Il est possible que la longévité observée des groupes soit nominale. Mais comment rendre compte, alors, du vieillissement tendanciel des effectifs ? Par ailleurs, l'instabilité en quelque sorte naturelle que l'on attribue aux bandes juvéniles, et par extension aux groupes criminels, tient précisément à leur incapacité présumée de perdurer comme groupe. 
ou parce que d'autres sortes de renseignements ne sont pas encore disponibles (niveau ou nature des revenus criminels des divers groupes ou index de «popularité culturelle» du style de vie pratiqué par les motards). L'objectif de cette étude n'était pas de proposer une vérification empirique d'une théorie en particulier mais de soumettre à la sociologie du milieu criminel un objet d'étude qu'elle a négligé.

\section{RÉFÉRENCES}

BLUMSTEIN, A., COHEN, J., ROTH, J.A., VISHER, C.A. eds. (1986), Criminal Careers and "Career Criminals", Washington D.C.: National Academy Press.

CHAIKEN, J.M. (1980), Models Used in Estimating Crime Rates, Appendix C. in Peterson M., Braiken, H., Doing Crime : A Survey of California Prison Inmates, Santa Monica, Calif.: Rand Corp.

COMMISSION D'ENQUÊTE SUR LE CRIME ORGANISÉ (1980), Les bandes de motards au Québec, Québec : Éditeur officiel du Québec.

CORDEAU, G. (1989), Les mécanismes de la dissuasion endogène, Revue canadienne de criminologie, 31, 3, 253-280.

CURRY, G.D., SPERGEL, I.A. (1988), Gang Homicide, Delinquency and Community, Criminology, 26, 3, 381-405.

FARRINGTON, D.P. (1986), Age and Crime, in M. Tonry and N. Morris (eds), Crime and Justice, Chicago, University of Chicago Press, 189-250.

GURR, T.R. (1977), Crime Trends in Modem Democracies Since 1945, Annales internationales de criminologie, 16, 2, 41-85.

HOROWITZ, R. (1982), Adult Delinquent Gangs in a Chicago Community, Urban Life, $11,1,3-26$.

JACOB, J.B. (1974), Street Gangs Behind Bars, Social Problems, 21, 395-408.

JACOB, J.B. (1976), Stratification and Conflict Among Prison Inmates, Journal of Criminal Law and Criminology, 66, 4, 476-482.

JACOB, J.B. (1977), Stateville : The Penitentiary in Mass Society, Chicago, University of Chicago Press.

JACOB, J.B. (1979), Race Relations and The Prisoners Subculture, in M. Tonry et N. Morris (eds), Crime and Justice (vol. 1), Chicago, University of Chicago Press, $1-28$.

KLEIN, W. and CRAWFORD, L.Y. (1967), Groups, Gangs and Cohesiveness, Journal of Research in Crime and Delinquency, 4, 142-165.

LEBLANC, M. (1986), La carrière criminelle : définition et prédiction, Criminologie, XIX, 2, 79-100.

LOEBER, R., STOUTHAMER-LOEBER. M. (1986), La prédiction de la délinquance, Criminologie, XIX, 2, 49-78.

LOWE, M. (1988), Conspiracy of Brothers, McClelland-Bantam, Toronto.

MONTGOMERY, R. (1976), The Outlaw Motorcycle Subculture, Revue canadienne de criminologie, 18, 3, 245-257. 
MONTGOMERY, R. (1977), The Outlaw Motorcycle Subculture (suite), Revue canadienne de criminologie, 19, 4, 356-361.

PETERSILIA, J., GREENWOOD, P.W., LAVIN, M. (1977), Criminal Careers of Habitual Felons, Santa Monica, Calif.: Rand Corporation.

PETERSON, M.A., BRAIKER, H.B. (1980), Doing Crime: A Survey of California Prison Inmates, Santa Monica, Calif.: Rand Corporation.

REISS, A. J. Jr. (1980), Understanding changes in crime rates, in S.E. Fienberg and A.J. Reiss Jr. (eds), Indicators of Crime and Criminal Justice: Quantitative Studies, Washington, D.C.: Bureau of Justice Statistics.

REISS, A.J. (1986), Co-Offender Influences on Criminal Careers, in A. Blumstein, J. Cohen, J.A. Roth, C.A. Visher (eds), Criminal Careers and "Career Criminals", National Academy Press, Washington D.C., vol. 2, 121-160.

REUTER, P. (1983), Disorganized Crime, Boston, M.I.T. Press.

SARNECKI, J. (1986), Delinquent Networks, Stockholm, National Council for Crime Prevention Sweden.

SIMON, F. (1971), Prediction Methods in Criminology, Londres: HMSO.

SINGER, S.I. (1981), Homogenous Victim-Offender Population: A Review and Some Research Implications, Journal of Criminal Law and Criminology, 72: 779-788.

STAFFORD, M. (1986), Gang Delinquency in Meiers (ed.), Major Forms of Crime, Beverly Hills, Sage, 167-190.

SUTTLES, G.D. (1968), The Social Order of the Slum, Chicago, Ill.: University of Chicago Press.

THOMPSON, H.S. (1968), Hell's Angels, Random House, New York.

WOLFGANG, M.E., FIGLIO, R.M., SELLIN, T.E. (1972), Delinquency in a Birth Cohort, Chicago, University of Chicago Press.

YABLONSKY, L. (1963), The Violent Gang, New York, MacMillan. 
ANNEXE: COORDONNÉES DES GROUPES DE MOTARDS RETENUS POUR ANALYSE

\begin{tabular}{|c|c|c|c|c|c|c|c|c|c|c|c|c|}
\hline \multirow[b]{2}{*}{ Nom du groupe } & \multicolumn{12}{|c|}{ Âge moyen des membres fichés par groupe et par année d'observation } \\
\hline & $\begin{array}{l}\text { Durée } \\
\text { d'opération } \\
\text { du groupe }\end{array}$ & $\begin{array}{l}\text { Nombre } \\
\text { d'individus } \\
\text { fichés par } \\
\text { groupe }\end{array}$ & 1974 & 1975 & 1977 & 1978 & 1980 & 1982 & 1984 & 1985 & 1988 & $\begin{array}{c}\text { Localisation } \\
\text { du groupe }\end{array}$ \\
\hline Aigles du Québec & $1969 \rightarrow$ & 53 & & & & & & $\begin{array}{l}N=17 \\
X=20\end{array}$ & $\begin{array}{l}N=3 \\
X=22\end{array}$ & & & Québec \\
\hline Atlans & $1971 \rightarrow$ & 12 & & & $\begin{array}{l}N=6 \\
X=22\end{array}$ & & & & & & & Grande Vallée \\
\hline Atomes** & $\begin{array}{l}1970-1983 \\
1970 \rightarrow\end{array}$ & 120 & $\begin{array}{l}N=34 \\
X=22\end{array}$ & $\begin{array}{l}\mathrm{N}=31 \\
\mathrm{X}=23\end{array}$ & $\begin{array}{l}N=52 \\
X=25\end{array}$ & $\begin{array}{l}\mathrm{N}=29 \\
\mathrm{X}=24.5\end{array}$ & $\begin{array}{l}N=31 \\
X=25\end{array}$ & $\begin{array}{l}\mathrm{N}=24 \\
\mathrm{X}=27.5\end{array}$ & $\begin{array}{l}N=11 \\
X=29.5\end{array}$ & & & Sherbrooke \\
\hline Béliers & $1980-1982$ & 9 & & & & & & $\begin{array}{l}\mathrm{N}=9 \\
\mathrm{X}=22.5\end{array}$ & & & & Châteauguay \\
\hline Blacks & $1980-1983$ & 14 & & & & & & $\begin{array}{l}N=14 \\
X=26.5\end{array}$ & & & & $\begin{array}{l}\text { Saint-Joseph-de-Beauce } \\
\text { Senneterre }\end{array}$ \\
\hline Black Eagle & $1978 ?$ & 25 & & & & & & & & & & Montréal-Gatineau \\
\hline Black Lake & (?) & 15 & & & $\begin{array}{l}N=10 \\
X=18.5\end{array}$ & & & & & & & \\
\hline Black Spiders & $1971-1979$ & 32 & $\begin{array}{l}N=3 \\
X=20\end{array}$ & & $\begin{array}{l}N=17 \\
X=22\end{array}$ & $\begin{array}{l}\mathrm{N}=12 \\
\mathrm{X}=23.5\end{array}$ & & & & & & $\begin{array}{l}\text { Montmagny } \\
\text { Saint-Michel-de- } \\
\text { Bellechasse Saint-Pascal }\end{array}$ \\
\hline
\end{tabular}




\begin{tabular}{|c|c|c|c|c|c|c|c|c|c|c|c|c|}
\hline \multirow[b]{2}{*}{ Nom du groupe } & \multirow[b]{2}{*}{$\begin{array}{l}\text { Durée } \\
\text { d'opération } \\
\text { du groupe }\end{array}$} & \multicolumn{10}{|c|}{ Âge moyen des membres fichés par groupe et par année d'observation } & \multirow[b]{2}{*}{$\begin{array}{l}\text { Localisation } \\
\text { du groupe }\end{array}$} \\
\hline & & $\begin{array}{l}\text { Nombre } \\
\text { d'individus } \\
\text { fichés par } \\
\text { groupe }\end{array}$ & 1974 & 1975 & 1977 & 1978 & 1980 & 1982 & 1984 & 1985 & 1988 & \\
\hline Blatnois & $1970 \rightarrow$ & 22 & & & & $\begin{array}{l}N=8 \\
X=23\end{array}$ & $\begin{array}{l}N=11 \\
X=25\end{array}$ & $\begin{array}{l}N=10 \\
X=27\end{array}$ & $\begin{array}{l}N=7 \\
X=29\end{array}$ & $\begin{array}{l}N=7 \\
X=30\end{array}$ & & Saint-Wenceslas \\
\hline Branded & $1977-1978$ & 5 & & & & $\begin{array}{l}N=5 \\
X=33\end{array}$ & & & & & & \\
\hline Cent Noms & $1972 \rightarrow$ & 65 & & & & & $\begin{array}{l}N=6 \\
X=32\end{array}$ & $\begin{array}{l}N=6 \\
X=34\end{array}$ & $\begin{array}{l}\mathrm{N}=6 \\
\mathrm{X}=36\end{array}$ & $\begin{array}{l}N=7 \\
X=34\end{array}$ & & Shawinigan \\
\hline Christ Drivers & $1972 \rightarrow$ & 12 & & $\begin{array}{l}N=4 \\
X=27\end{array}$ & $\begin{array}{l}N=4 \\
X=29\end{array}$ & $\begin{array}{l}N=6 \\
X=28\end{array}$ & $\begin{array}{l}N=4 \\
X=32\end{array}$ & $\begin{array}{l}N=10 \\
X=31\end{array}$ & & & & Senneterre \\
\hline Comanches & $1970-1980$ & 12 & & & $\begin{array}{l}\mathrm{N}=12 \\
\mathrm{X}=23.5\end{array}$ & & & & & & & Valleyfield \\
\hline Conquatcheros & $1971-1986$ & 13 & & $\begin{array}{l}N=1 \\
X=28\end{array}$ & & & $\begin{array}{l}N=4 \\
X=26\end{array}$ & $\begin{array}{l}N=2 \\
X=29\end{array}$ & & & & $\begin{array}{l}\text { Sacré-Coeur, Alma } \\
\text { Chicoutimi, Roberval, } \\
\text { Port Alfred }\end{array}$ \\
\hline Cyclones & $1971-1980$ & 37 & $\begin{array}{l}N=2 \\
X=31\end{array}$ & $\begin{array}{l}N=2 \\
X=32\end{array}$ & $\begin{array}{l}\mathrm{N}=36 \\
\mathrm{X}=26.5\end{array}$ & & & & & & & $\begin{array}{l}\text { Montréal } \\
\text { Magog }\end{array}$ \\
\hline Death Riders & $1978 \rightarrow$ & 58 & & & & $\begin{array}{l}\mathrm{N}=8 \\
\mathrm{X}=23.5\end{array}$ & $\begin{array}{l}N=2 \\
X=24\end{array}$ & $\begin{array}{l}N=7 \\
X=30\end{array}$ & $\begin{array}{l}N=21 \\
X=31\end{array}$ & $\begin{array}{l}\mathrm{N}=23 \\
\mathrm{X}=30.5\end{array}$ & & Laval \\
\hline
\end{tabular}


Âge moyen des membres fichés par groupe et par année d'observation

\begin{tabular}{|c|c|c|c|c|c|c|c|c|c|c|c|c|}
\hline Nom du groupe & $\begin{array}{l}\text { Durée } \\
\text { d'opération } \\
\text { du groupe }\end{array}$ & $\begin{array}{l}\text { Nombre } \\
\text { d'individus } \\
\text { fichés par } \\
\text { groupe }\end{array}$ & 1974 & 1975 & 1977 & 1978 & 1980 & 1982 & 1984 & 1985 & 1988 & $\begin{array}{l}\text { Localisation } \\
\text { du groupe }\end{array}$ \\
\hline Devils Disciples* & $1968-1976$ & 10 & & $\begin{array}{l}N=7 \\
X=28\end{array}$ & $\begin{array}{l}N=2 \\
X=31\end{array}$ & $\begin{array}{l}\mathrm{N}=2 \\
\mathrm{X}=32\end{array}$ & $\begin{array}{l}N=2 \\
X=34\end{array}$ & & & & & Montréal \\
\hline El Toros & $1980-1988$ & 13 & & & & & & & $\begin{array}{l}N=12 \\
X=23\end{array}$ & $\begin{array}{l}N=1 \\
X=21\end{array}$ & $\begin{array}{l}N=2 \\
X=24\end{array}$ & Saint-Prosper-de-Beauce \\
\hline Escape Hell & 1974-1979? & 2 & & $\begin{array}{l}N=2 \\
X=25\end{array}$ & & & & & & & & \\
\hline Evils One & 1981 & 17 & & & & & & $\begin{array}{l}N=1 \\
X=20\end{array}$ & $\begin{array}{l}N=1 \\
X=22\end{array}$ & $\begin{array}{l}N=7 \\
X=28\end{array}$ & $\begin{array}{l}N=8 \\
X=27\end{array}$ & $\begin{array}{l}\text { Saint-Basile } \\
\text { Victoriaville } \\
\text { Drummondville }\end{array}$ \\
\hline Executeurs* & $1980-1982$ & 61 & & & & & $\begin{array}{l}N=35 \\
X=19\end{array}$ & $\begin{array}{l}N=18 \\
X=20\end{array}$ & $\begin{array}{l}N=12 \\
X=21.5\end{array}$ & $\begin{array}{l}N=12 \\
X=22.5\end{array}$ & $\begin{array}{l}N=12 \\
X=25.5\end{array}$ & Montréal \\
\hline Eyes Stone & $1974 \rightarrow$ & 23 & & & $\begin{array}{l}N=15 \\
X=23\end{array}$ & $\begin{array}{l}N=15 \\
X=24\end{array}$ & $\begin{array}{l}N=15 \\
X=26\end{array}$ & $\begin{array}{l}\mathrm{N}=16 \\
\mathrm{X}=26.5\end{array}$ & $\begin{array}{l}\mathrm{N}=16 \\
\mathrm{X}=28.5\end{array}$ & $\begin{array}{l}N=19 \\
X=29.5\end{array}$ & $\begin{array}{l}N=20 \\
X=35\end{array}$ & Saint-Prime \\
\hline Flambeurs & $1972-1980$ & 6 & & & & & & & & & & Mont-Joli \\
\hline Gitans & $1970-1984$ & 79 & $\begin{array}{l}N=30 \\
X=23\end{array}$ & $\begin{array}{l}N=6 \\
X=24\end{array}$ & $\begin{array}{l}N=24 \\
X=26\end{array}$ & $\begin{array}{l}N=17 \\
X=24\end{array}$ & $\begin{array}{l}N=13 \\
X=28\end{array}$ & & & & & Sherbrooke \\
\hline
\end{tabular}




\begin{tabular}{|c|c|c|c|c|c|c|c|c|c|c|c|c|}
\hline \multirow[b]{2}{*}{ Nom du groupe } & \multirow[b]{2}{*}{$\begin{array}{l}\text { Durée } \\
\text { d'opération } \\
\text { du groupe }\end{array}$} & \multicolumn{10}{|c|}{ Âge moyen des membres fichés par groupe et par année d'observation } & \multirow[b]{2}{*}{$\begin{array}{l}\text { Localisation } \\
\text { du groupe }\end{array}$} \\
\hline & & $\begin{array}{l}\text { Nombre } \\
\text { d'individus } \\
\text { fichés par } \\
\text { groupe }\end{array}$ & 1974 & 1975 & 1977 & 1978 & 1980 & 1982 & 1984 & 1985 & 1988 & \\
\hline Groupe des dix & $1983-1985$ & 15 & & & & & & & & $\begin{array}{l}N=8 \\
X=28\end{array}$ & & Drummondville \\
\hline Hell's Angels & $1977 \rightarrow$ & 190 & & & $\begin{array}{l}\mathrm{N}=10 \\
X=26.5\end{array}$ & $\begin{array}{l}\mathrm{N}=53 \\
\mathrm{X}=27.5\end{array}$ & $\begin{array}{l}N=43 \\
X=29\end{array}$ & $\begin{array}{l}N=21 \\
X=31\end{array}$ & $\begin{array}{l}\mathrm{N}=24 \\
\mathrm{X}=32.5\end{array}$ & $\begin{array}{l}N=22 \\
X=34.5\end{array}$ & $\begin{array}{l}N=4 \\
X=39\end{array}$ & $\begin{array}{l}\text { Sorel, Lennoxville } \\
\text { Montréal, Laval }\end{array}$ \\
\hline Homets & 1971- ? & 3 & $\begin{array}{l}N=3 \\
X=20\end{array}$ & & & & & & & & & Sherbrooke \\
\hline Huns & $1973-1983$ & 4 & & & & $\begin{array}{l}N=1 \\
X=25\end{array}$ & $\begin{array}{l}N=1 \\
X=28\end{array}$ & & & & & Rosemère \\
\hline Incas* & $1972-1978$ & 12 & & & & & $\begin{array}{l}\mathrm{N}=3 \\
\mathrm{X}=21.5\end{array}$ & $\begin{array}{l}N=11 \\
X=27\end{array}$ & & & & $\begin{array}{l}\text { Amos } \\
\text { Val d'Or }\end{array}$ \\
\hline Incompris & $1974-1978$ & 2 & $\begin{array}{l}N=2 \\
X=24\end{array}$ & & & & & & & & & \\
\hline Inconnus & $1977-1985$ & 38 & & & & $\begin{array}{l}\mathrm{N}=24 \\
\mathrm{X}=18.5\end{array}$ & & $\begin{array}{l}N=8 \\
X=24\end{array}$ & $\begin{array}{l}N=3 \\
X=37\end{array}$ & & & Mascouche \\
\hline Indépendants & $1976-1981$ & 4 & & & & & $\begin{array}{l}N=2 \\
X=24.5\end{array}$ & & & & & Saint-Jean-Chrysostome \\
\hline
\end{tabular}


Âge moyen des membres fichés par groupe et par année d'observation

\begin{tabular}{|c|c|c|c|c|c|c|c|c|c|c|c|}
\hline Nom du groupe & $\begin{array}{l}\text { Durée } \\
\text { d'opération } \\
\text { du groupe }\end{array}$ & $\begin{array}{l}\text { Nombre } 1974 \\
\text { d'individus } \\
\text { fichés par } \\
\text { groupe }\end{array}$ & 1975 & 1977 & 1978 & 1980 & 1982 & 1984 & 1985 & 1988 & $\begin{array}{l}\text { Localisation } \\
\text { du groupe }\end{array}$ \\
\hline Indésirables* & $1970-1978$ & 16 & & & & & $\begin{array}{l}N=5 \\
X=20\end{array}$ & $\begin{array}{l}N=1 \\
X=22\end{array}$ & & & Saint-Lin \\
\hline Iron Coffin & $1979 \rightarrow$ & 8 & & & & $\begin{array}{l}\mathrm{N}=2 \\
\mathrm{X}=25\end{array}$ & $\begin{array}{l}\mathrm{N}=4 \\
\mathrm{X}=27\end{array}$ & $\begin{array}{l}N=4 \\
X=29\end{array}$ & $\begin{array}{l}N=4 \\
X=30\end{array}$ & $\begin{array}{l}N=5 \\
X=34\end{array}$ & La Pocatière \\
\hline Liberty Angels & $?$ & 4 & & & $\begin{array}{l}N=4 \\
X=23\end{array}$ & & & & & & \\
\hline Maraudeurs & $1974-1980$ & 20 & & & $\begin{array}{l}N=8 \\
X=22\end{array}$ & $\begin{array}{l}N=11 \\
X=25.5\end{array}$ & & & & & Asbestos \\
\hline Mercenaires & $1972 \rightarrow$ & 35 & & & $\begin{array}{l}N=3 \\
X=22.5\end{array}$ & $\begin{array}{l}N=22 \\
X=25\end{array}$ & & $\begin{array}{l}N=17 \\
X=28\end{array}$ & $\begin{array}{l}\mathrm{N}=20 \\
\mathrm{X}=28.5\end{array}$ & & $\begin{array}{l}\text { Lac Mégantic } \\
\text { Saint-Prosper-de-Beauce }\end{array}$ \\
\hline Merciless Riders & $1981-1988$ & 13 & & & & & & $\begin{array}{l}N=8 \\
X=29\end{array}$ & & & $\begin{array}{l}\text { Montréal } \\
\text { Laval }\end{array}$ \\
\hline Mirages & $1981 \rightarrow$ & 25 & & & & & $\begin{array}{l}N=1 \\
X=24\end{array}$ & $\begin{array}{l}N=15 \\
X=27\end{array}$ & $\begin{array}{l}N=1 \\
X=29\end{array}$ & & $\begin{array}{l}\text { Saint-Janvier } \\
\text { Laval }\end{array}$ \\
\hline Missiles & $1970-1982$ & 50 & & & $\begin{array}{l}N=17 \\
X=26\end{array}$ & $\begin{array}{l}N=1 \\
X=24\end{array}$ & & & $\begin{array}{l}N=1 \\
X=30\end{array}$ & & $\begin{array}{l}\text { Baie Comeau } \\
\text { Saint-Gédéon, Saguenay }\end{array}$ \\
\hline New Generation & $1971-1981$ & 6 & & & $\begin{array}{l}N=6 \\
X=26\end{array}$ & & & & & & Havre Saint-Pierre \\
\hline
\end{tabular}


Âge moyen des membres fichés par groupe et par année d'observation

\begin{tabular}{|c|c|c|c|c|c|c|c|c|c|c|c|c|}
\hline Nom du groupe & $\begin{array}{l}\text { Durée } \\
\text { d'opération } \\
\text { du groupe }\end{array}$ & $\begin{array}{l}\text { Nombre } \\
\text { d'individus } \\
\text { fichés par } \\
\text { groupe }\end{array}$ & 1974 & 1975 & 1977 & 1978 & 1980 & 1982 & 1984 & 1985 & 1988 & $\begin{array}{c}\text { Localisation } \\
\text { du groupe }\end{array}$ \\
\hline Night Angels & $1972-1983$ & 13 & & & & $\begin{array}{l}\mathrm{N}=12 \\
\mathrm{X}=24.5\end{array}$ & $\begin{array}{l}N=1 \\
X=27\end{array}$ & & & & & Sept-Iles \\
\hline Nomades & $1969-1980$ & 35 & & & $\begin{array}{l}N=2 \\
X=25.5\end{array}$ & $\begin{array}{l}N=7 \\
X=24\end{array}$ & $\begin{array}{l}N=4 \\
x=26\end{array}$ & & & & & Valleyfield \\
\hline Outlaws & $1977 \rightarrow$ & 100 & & & $\begin{array}{l}N=16 \\
X=29\end{array}$ & $\begin{array}{l}N=22 \\
X=25.5\end{array}$ & $\begin{array}{l}N=24 \\
X=29\end{array}$ & $\begin{array}{l}N=13 \\
X=29\end{array}$ & $\begin{array}{l}N=15 \\
X=30\end{array}$ & $\begin{array}{l}N=18 \\
X=31\end{array}$ & $\begin{array}{l}\mathrm{N}=21 \\
\mathrm{X}=32.5\end{array}$ & $\begin{array}{l}\text { Danville, Montréal, } \\
5 \text { Joliette }\end{array}$ \\
\hline Pacific Rebels & $1970-1980$ & 102 & $\begin{array}{l}N=24 \\
X=26\end{array}$ & $\begin{array}{l}N=1 \\
X=30\end{array}$ & $\begin{array}{l}\mathrm{N}=33 \\
\mathrm{X}=25\end{array}$ & & $\begin{array}{l}N=1 \\
X=27\end{array}$ & & & & & Québec \\
\hline Popeyes & $1968-1970$ & 47 & & $\begin{array}{l}\mathrm{N}=6 \\
\mathrm{X}=23.5\end{array}$ & & & & & & & & $\begin{array}{l}\text { Sorel } \\
\text { Montréal }\end{array}$ \\
\hline Prides & 1978-1981 & 16 & & & & & $\begin{array}{l}\mathrm{N}=9 \\
\mathrm{X}=20\end{array}$ & & & & & Shawinigan \\
\hline Primitifs & $1971 \rightarrow$ & 75 & & & $\begin{array}{l}N=6 \\
X=24\end{array}$ & $\begin{array}{l}\mathrm{N}=7 \\
\mathrm{X}=28\end{array}$ & $\begin{array}{l}N=6 \\
X=27\end{array}$ & $\begin{array}{l}N=6 \\
X=29\end{array}$ & $\begin{array}{l}N=15 \\
X=32\end{array}$ & & & $\begin{array}{l}\text { Beauharnois } \\
\text { Sainte-Catherine }\end{array}$ \\
\hline Québécois & $1980-1983$ & 10 & & & $\begin{array}{l}N=6 \\
X=23\end{array}$ & $\begin{array}{l}\mathrm{N}=4 \\
\mathrm{X}=23.5\end{array}$ & $\begin{array}{l}N=8 \\
X=24.5\end{array}$ & & & & & Coaticook \\
\hline Quidams & $1970-1984$ & 41 & & & $\begin{array}{l}\mathrm{N}=31 \\
\mathrm{X}=22.5\end{array}$ & $\begin{array}{l}N=13 \\
X=27\end{array}$ & $\begin{array}{l}\mathrm{N}=13 \\
\mathrm{X}=27.5\end{array}$ & $\begin{array}{l}\mathrm{N}=4 \\
\mathrm{X}=32.5\end{array}$ & $\begin{array}{l}N=1 \\
X=33\end{array}$ & $\begin{array}{l}N=1 \\
X=27\end{array}$ & & $\begin{array}{l}\text { Saint-Ours } \\
\text { Danville }\end{array}$ \\
\hline
\end{tabular}




\begin{tabular}{|c|c|c|c|c|c|c|c|c|c|c|c|c|}
\hline \multirow[b]{2}{*}{ Nom du groupe } & \multicolumn{12}{|c|}{ Âge moyen des membres fichés par groupe et par année d'observation } \\
\hline & $\begin{array}{l}\text { Durée } \\
\text { d'opération } \\
\text { du groupe }\end{array}$ & $\begin{array}{l}\text { Nombre } \\
\text { d'individus } \\
\text { fichés par } \\
\text { groupe }\end{array}$ & 1974 & 1975 & 1977 & 1978 & 1980 & 1982 & 1984 & 1985 & 1988 & $\begin{array}{l}\text { Localisation } \\
\text { du groupe }\end{array}$ \\
\hline Ravagers & $?$ & 3 & & & & & $\begin{array}{l}N=3 \\
X=23\end{array}$ & & & & & \\
\hline Rebels & $1975-1980$ & 35 & $\begin{array}{l}N=17 \\
X=24.5\end{array}$ & & & & $\begin{array}{l}N=15 \\
X=25.5\end{array}$ & & & & & $\begin{array}{l}\text { Greenbay } \\
\text { Windsor }\end{array}$ \\
\hline Réincarnés & $1981 \rightarrow$ & 20 & & & & & & $\begin{array}{l}N=16 \\
X=26.5\end{array}$ & & & & Trois-Rivières \\
\hline Renegates & $1980-1986$ & 18 & & & & $\begin{array}{l}N=2 \\
X=26\end{array}$ & $\begin{array}{l}N=5 \\
X=27\end{array}$ & $\begin{array}{l}N=5 \\
X=29\end{array}$ & & $\begin{array}{l}N=15 \\
X=32\end{array}$ & & Montréal \\
\hline Rolling Eagle & $1971-1984$ & 12 & & & & & & & $\begin{array}{l}\mathrm{N}=12 \\
\mathrm{X}=28.5\end{array}$ & & & Saint-Apolinaire \\
\hline Satan's Choice & $1968-1976$ & 13 & & $\begin{array}{l}\mathrm{N}=1 \\
\mathrm{X}=25\end{array}$ & & & & & & & & Montréal \\
\hline Sex Fox & $1971-1980$ & 33 & & & $\begin{array}{l}\mathrm{N}=22 \\
\mathrm{X}=23.5\end{array}$ & $\begin{array}{l}N=31 \\
X=24\end{array}$ & & & & & & Chibougamau \\
\hline Sky Trippers & ¿ 1983-1985? & 10 & & & & & & & $\begin{array}{l}N=1 \\
X=22\end{array}$ & $\begin{array}{l}N=1 \\
X=23\end{array}$ & & Montréal \\
\hline Society Rejet & $1970-1975$ & 6 & & & & & & & & & & Montréal \\
\hline
\end{tabular}




\begin{tabular}{|c|c|c|c|c|c|c|c|c|c|c|c|c|}
\hline \multirow[b]{2}{*}{ Nom du groupe } & \multirow[b]{2}{*}{$\begin{array}{l}\text { Durée } \\
\text { d'opération } \\
\text { du groupe }\end{array}$} & \multicolumn{10}{|c|}{ Âge moyen des membres fichés par groupe et par année d'observation } & \multirow[b]{2}{*}{$\begin{array}{l}\text { Localisation } \\
\text { du groupe }\end{array}$} \\
\hline & & $\begin{array}{l}\text { Nombre } \\
\text { d'individus } \\
\text { fichés par } \\
\text { grroupe }\end{array}$ & 1974 & 1975 & 1977 & 1978 & 1980 & 1982 & 1984 & 1985 & 1988 & \\
\hline S.S. & $1980-1985$ & 48 & & & & & & $\begin{array}{l}N=35 \\
X=27.5\end{array}$ & & & & Montréal \\
\hline Union $\mathrm{X}$ & $1971-1984$ & 3 & & $\begin{array}{l}N=2 \\
X=33\end{array}$ & & & & & & & & $\begin{array}{l}\text { Sainte-Croix } \\
\text { Lotbinière }\end{array}$ \\
\hline Vampires & $1977-1980$ & 7 & & & & $\begin{array}{l}N=3 \\
X=22.5\end{array}$ & $\begin{array}{l}N=1 \\
X=26\end{array}$ & & & & & Beloeil \\
\hline Vickings & $1971-1986$ & 31 & & & $\begin{array}{l}N=6 \\
X=24\end{array}$ & $\begin{array}{l}N=4 \\
X=25\end{array}$ & $\begin{array}{l}N=4 \\
X=27\end{array}$ & $\begin{array}{l}N=12 \\
X=7.5\end{array}$ & $\begin{array}{l}N=7 \\
X=29\end{array}$ & $\begin{array}{l}N=15 \\
X=30\end{array}$ & & $\begin{array}{l}\text { Matane } \\
\text { Granby }\end{array}$ \\
\hline Warriors & 1984-1988 & 13 & & & & & & & $\begin{array}{l}\mathbf{N}=11 \\
\mathrm{X}=\mathbf{2 7 . 5}\end{array}$ & & & Boisbriand \\
\hline Z'Beers & $1979-1984$ & 22 & & & & & & $\begin{array}{l}N=18 \\
X=25\end{array}$ & & & & Saint-Hyacinthe \\
\hline
\end{tabular}

* Les observations relevées après la dissolution du groupe concernent les membres ayant encore une activité délinquante en relation avec d'autres groupes actifs, mais revendiquant leur statut d'ex-membres.

** Un problème de concordance entre les données policières et les données de nos entretiens avec des ex-membres de groupes de motards empêche de définir avec précision le début et la fin du groupe. 